\title{
Measurement of neutron and charged particle fluxes toward earthquake prediction
}

\author{
Asatulla U. Maksudov • Mars A. Zufarov
}

Received: 27 December 2016/Accepted: 6 November 2017/Published online: 7 December 2017

(C) The Author(s) 2017. This article is an open access publication

\begin{abstract}
In this paper, we describe a possible method for predicting the earthquakes, which is based on simultaneous recording of the intensity of fluxes of neutrons and charged particles by detectors, commonly used in nuclear physics. These low-energy particles originate from radioactive nuclear processes in the Earth's crust. The variations in the particle flux intensity can be the precursor of the earthquake. A description is given of an electronic installation that records the fluxes of charged particles in the radial direction, which are a possible response to the accumulated tectonic stresses in the Earth's crust. The obtained results showed an increase in the intensity of the fluxes for 10 or more hours before the occurrence of the earthquake. The previous version of the installation was able to indicate for the possibility of an earthquake (Maksudov et al. in Instrum Exp Tech 58:130-131, 2015), but did not give information about the direction of the epicenter location. In this regard, the installation was modified by adding eight directional detectors. With the upgraded setup, we have received both the predictive signals, and signals determining the directions of the location of the forthcoming earthquake, starting 2-3 days before its origin.
\end{abstract}

Keywords Detector - Neutron flux - Charged particles · Radioactive decay $\cdot$ Earthquake

A. U. Maksudov $(\bowtie) \cdot$ M. A. Zufarov

Physical - Technical Institute Academy of Sciences of Uzbekistan, 2-b, Bodomzor Str., Tashkent, Uzbekistan 100084

e-mail: asaduz50@rambler.ru

\section{Introduction}

The earthquakes occur suddenly, and substantial movements of the ground lead to destruction of buildings and other constructions. As a result, serious harm to industry is inflicted due to destruction of underground communication systems, and most importantly, large numbers of population become victims of the disaster. Recent examples may include the series of earthquakes, happened in Honshu, Japan, March 11, 2011, with magnitude $M_{\mathrm{W}}$ 9.1; L'Aquila, Italy, April 6, 2009, $M_{\mathrm{W}} 6.3$; Sichuan, China, May 12, 2008, $M_{\mathrm{S}}$ 8.0; Kashmir, Pakistan, October 8, 2005, $M_{\mathrm{W}} 7.6$; Sumatra-Andaman, Indonesia, December 26, 2004, $M_{\mathrm{W}} 9.1$; Izmit, Turkey, August 17, 1999, $M_{\mathrm{W}}$ 7.6, etc.

Attempts to predict earthquakes began long ago, using different methods and analyzing data from numerous grids of seismic stations. All over the world, scientists continue to look for new precursors of seismic events. In large areas around the epicenter of the forthcoming earthquake, the precursors are detected in different geophysical parameters. Nowadays, nearly 200 precursors are known, which are used separately or in combination, in attempts to develop a method for predicting the earthquake. Almost all methods of monitoring and forecasting the earthquakes deal with physical effects on the Earth's surface, such as thermal anomalies, magnetic disturbances, radio signal anomalies, ionospheric and sub-ionospheric signal propagation disorders, electron density fluctuations (Hazra et al. 2015, 2017; Setyaningsih et al. 2014; Zeren et al. 2014; Silva et al. 2013; Karia et al. 2013; Li and Parrot 2013; Sarkar et al. 2011, 2012; De et al. 2010, 2011a, b, 2012).

Presently, the world seismic service is engaged in analyzing the earthquakes only post-factum. So far the problem in traditional seismology consists in identifying the 
forthcoming catastrophic earthquake, localized in space and time, from the huge amount of data provided by seismic detectors. However, the progress in this direction has been limited, and up to date, a quantitative and reliable method of predicting the earthquake of any time span, long term or short term, does not exist.

Nowadays, great efforts are being invested toward solution of the earthquake prediction problem. One of the recent conceptions is based on simultaneous monitoring of several factors, responsible for the earthquake. The objective of this work is to reveal the link between variations in the intensity of fluxes of neutrons and charged particles, emanating from the Earth's crust, with the forthcoming earthquake. Besides, we examine the possibility to specify the direction of the epicenter by means of additional detectors, surrounding the installation.

\section{Radon gas, low-energy neutrons and charged particles as earthquake precursors}

When emission of radioactive gases from the Earth occurs, usually emission of radon is assumed. Before the seismic event, significant amount of radon and its isotope thoron appears in underground water, near the Earth's surface and atmosphere. Radon is recognized as an optimal indicator of the forthcoming earthquake. Decrease in its concentration in the environment due to decay and dissemination is always compensated by new emission from the Earth, where radioactive transformation of elements takes place. The average content of radon in a given location is constant, and it is defined by the concentration of uranium (radium) in this place.

Evidence of a forthcoming earthquake, which can be detected from the dynamics of radon emission, starts 3-4 months before the seismic event and becomes especially clear in the last $1-2$ weeks prior to earthquake. A sharp increase in radon's concentration begins within 1.5-3 days before the strike in the vicinity of the epicenter. The strike occurs after passage of the maximum of radon concentration in time.

When the upper crust of Earth is sufficiently permeable and convective flow of gases is present, radon can emanate from the depth up to $200 \mathrm{~m}$. Despite low concentration of radon in these gas flows, it can be easily detected due to its radioactivity. After the well-known Tashkent earthquake (April 26, 1966, M 5.1) which had lasted several days, causing massive destruction to the capital city of Tashkent, Uzbek scientists made a discovery that prior to the earthquake the concentration of radioactive radon in underground water notably increases (Ulomov and Mavashev 1967). This work initiated extensive research on the radon gas as a precursor of earthquakes [see the recent review (Woith 2015)].

Radioactive gas radon and its isotopes, as earthquake precursors, have a "local" character. According to research conducted by several authors, for the earthquake of magnitude 5, the radial distance where this method gives good results is up to $50-70 \mathrm{~km}$ from the epicenter. At longer distances, registration of radioactive gases becomes difficult due to their low concentration.

In seismic processes involving gas emission, radioactive decay of gaseous elements releasing $\alpha$-particles, which subsequently interact with terrestrial matter, gives rise to neutrons and charged particles. Unlike the radon gas itself, these particles can propagate to very long distances. Hence, variations in the intensity of fluxes of low-energy neutrons and charged particles, emanating from the Earth's crust, can be considered as precursor of the earthquake.

Investigations on the variation in the neutron flux near the Earth's surface, conducted by researches of the Nuclear Physics Institute at Moscow State University, have revealed their link to dynamic processes in the Earth's crust. They performed simultaneous measurements of the neutron flux with detectors, used in nuclear geophysics, and seismic waves from the earthquakes, whose epicenters were located at distances from 120 to $900 \mathrm{~km}$. Measurements were conducted from March 1996 to May 1998 near the city Alma-Ata, in a pit of $100 \mathrm{~m}$ depth. At average count rate of 12.5 pulse/s, approximately one day before to the earthquake, the count rate has increased by $2.0-3.5$ times (Antonova et al. 2007). In this experiment, the existence of a correlation between the variation in the neutron flux near the surface and the magnitude of the earthquake was discovered.

Presently, there are solid evidences that dynamic processes linked to terrestrial deformations show up in variations in the fluxes of slow neutrons and charged particles (Volodichev et al. 2003; Kuzhevskij et al. 2003). Continuous monitoring of the intensity of these particle fluxes can be helpful in solving the problem of earthquake prediction.

\section{A setup for measuring the intensity of fluxes of neutrons and charged particles emitted from the Earth's crust}

We have proposed and developed a new approach for investigation of the precursors of earthquakes, which is based on simultaneous detection of variations in the intensity of fluxes of low-energy neutrons and charged particles, emitted from the Earth's crust (Maksudov et al. 2015). The setup is assembled on detectors, commonly employed in nuclear physics experiments. The installation is designed to consider the following problems: 
- Exploring the links between variations in the intensity of fluxes of neutrons and charged particles with earthquakes and comparing with other geophysical processes in the Earth's crust.

- Finding functional dependence for these links and developing a scientific approach for their practical applications, aimed at predicting the earthquakes.

- Exploring the correlations between the time profiles of flux intensities of charged particles, significance of their variations in time and the location of the earthquake.

- Studying the correlations between the data obtained by the proposed method and corresponding data of seismic stations, in order to develop a scientific approach for specifying the location of the possible earthquake.

- Developing a method for predicting the earthquake ahead of time by $10 \mathrm{~h}$ and earlier.

Presently, accumulation of statistical data from the installation is under way. The sketch of the original setup, which occupies an area of $1 \mathrm{~m}^{2}$, is illustrated in Fig. 1. It contains four Cherenkov counters (1), beneath of which a scintillator is situated. Below the scintillator, an absorber (3) is placed. Then come a second scintillator (2) and another absorber (3). In the basement, beneath the background shields made of hydrogen containing substance (paraffin), a set of neutron counters are placed.

For the design of the setup, shown in Fig. 1, the Cherenkov detectors were unable to reveal the direction, from which the charged particles were coming; therefore, fixing the direction of the earthquake epicenter was not possible. In order to solve this problem, we have modified the installation, which will be described in the following section.

The resolution of a scintillator detector is different from that of a Cherenkov detector of the same thickness, as it is specified by statistical fluctuations of ionization. Their advantages are small idle time, high amplification rate combined with very high resolution time $10^{-10} \mathrm{~s}$, linear dependence of the output signal from the energy of the

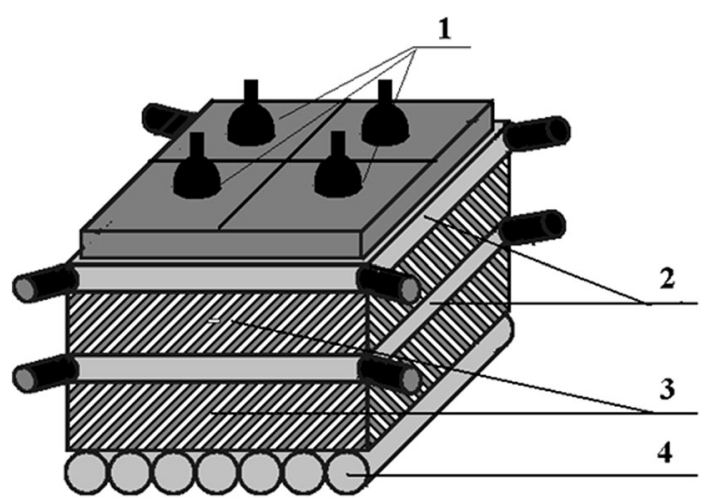

Fig. 1 -Cherenkov detectors. 2-Scintillator detectors. 3-Carbon absorbers. 4-Neutron counters incident particle. Fluctuations in the number of photoelectrons are negligible, as the light output from the scintillator is significantly greater.

The most part of the light, emitted by the scintillator, is created due to low-energy interactions. By contrast, in Cherenkov counters, light is emitted mainly by the particle itself, and the light output is proportional to energy of the incident charged particle, left in its sensitive region. For these reasons, we gave preference to scintillator detectors of directed action.

The experimental setup was built and installed in the basement room of the Physical-Technical Institute, Uzbek Academy of Sciences (Yuldashbaev and Maksudov 2010). Location of the setup in the basement space allows to reduce the cosmic ray contribution from the atmosphere by 2-3 times, thus minimizing the background component. Continuous measurements were performed. Around 20 earthquakes with magnitudes 3 and greater, which happened in Kyrgyzstan, Japan, Turkey, Tuva and some other places, were detected (Yuldashbaev and Maksudov 2012; Yuldashbaev et al. 2012). From the analysis of obtained results, we have concluded that signals from the detectors start to appear 2-3 days before the earthquakes. For these periods, the intensity of fluxes of neutrons and charged particles can increase by tens of times, which can suggest that short-time prediction of earthquakes by the proposed method is possible.

\section{Upgraded setup with directional detectors}

Another version of the setup for prediction of earthquakes with defining the direction of the epicenter was developed (Maksudov 2015, 2016). In this new design, the Cherenkov counters are excluded and the setup is surrounded from outer side by eight scintillator detectors, which are oriented in space and placed along octuple, as shown in Fig. 2. Four detectors are lined up with four parts of the world (north, east, south, west), and four others are placed between them. This electronic setup performs registration of signals in $2 \pi$ geometry. A particular detector, which records the greatest among registered signals, is expected to define the direction of the epicenter.

The scintillator detectors of direction are arranged along the perimeter of the setup, inclined at $45^{\circ}$ to the Earth's surface and $50 \mathrm{~cm}$ away from the edge of the installation. In this position, the greatest numbers of particles straightly fall to its surface. In addition, less internal reflections occur in the scintillator, and therefore, light absorption is reduced. All these factors contribute to maximal output signal from the photomultiplier. 


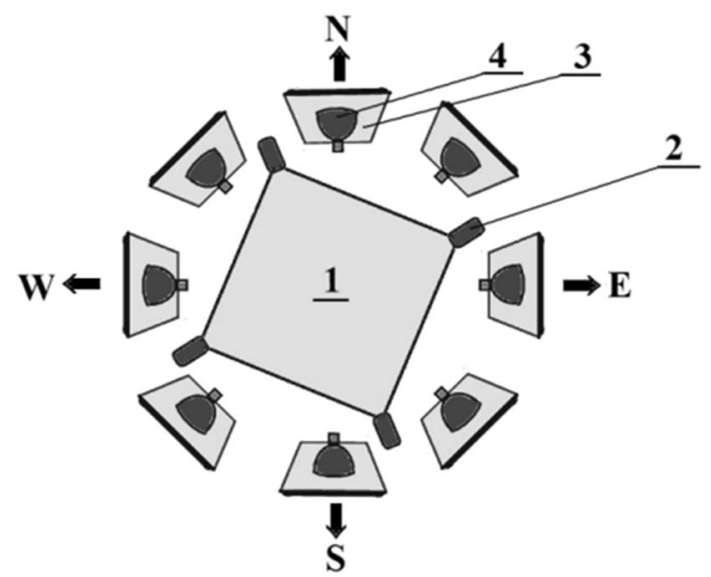

Fig. 2 Upgraded setup with detectors of direction (top view). $1-$ Electronic hybrid setup, 2-photomultiplier 84, 3-detectors of direction, 4-photomultiplier 125

The neutrons while slowing down in the absorber create particles, flying in the backward direction, so-called background of "rear" currents. To prevent these currents from being registered in the directional detectors, they are positioned at $50 \mathrm{~cm}$ distance from them. This distance is found to be sufficient for backward flying particles to avoid encountering with the directional detectors. The signals from particles flying "against" the detector are strongly reduced due to absorption in the black painted surface, and their contribution will be insignificant. This feature is responsible for the directional property of the detector.

Calibration measurement of light falling toward the detector and in opposite direction is illustrated in Fig. 3. As can be seen from this figure, the ratio of corresponding

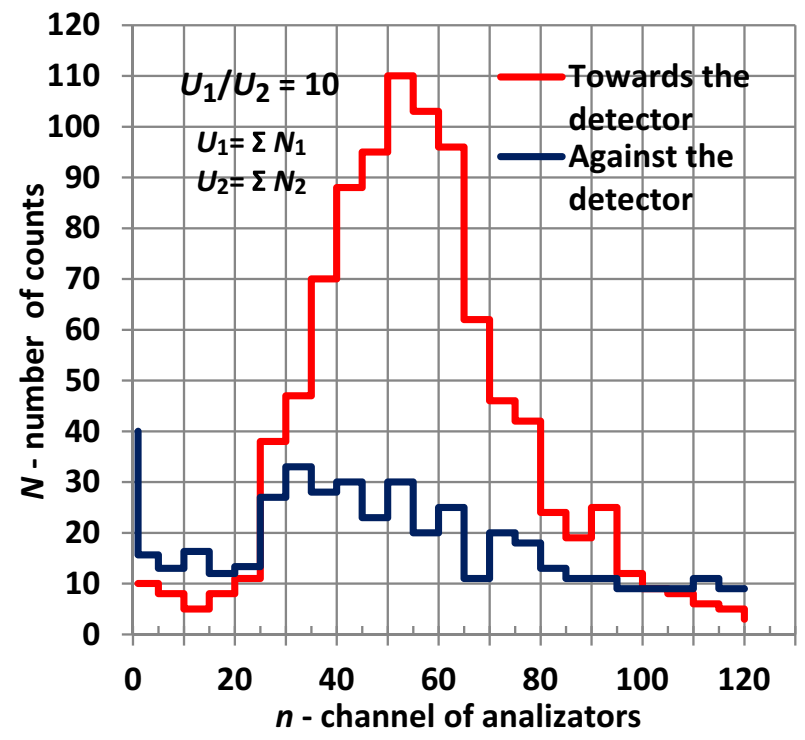

Fig. 3 Calibration measurement for the directional detector. The distribution of signals from the light, falling toward and against the detector cumulative signals is $\sim 10$, which allows to use this detector as a directional detector.

Testing measurements of the flux intensities of neutrons and charged particles have been carried out with the upgraded setup. Figure 4 shows the signal readings received from the prognostic detectors (electronic hybrid unit 1 in Fig. 2). These data indicate the occurrence of seismic activity in the Earth's crust, but cannot indicate a possible direction of this phenomenon. Therefore, we called these signals prognostic. Signals began to appear 3 days before the origin of the earthquake in Kyrgyzstan (July 27, 2016, $M$ 4.0), and the maximum signal was about 600 units. With the earthquake in Japan (August 21, 2016, $M$ 5.2), the signal appeared 2 days before its origin, and the signal intensity was about 450 units. At the same time, signals were recorded from the detectors of direction (eight surrounding detectors in Fig. 2).

Comparison with the data of the European-Mediterranean Seismological Center (http://www.emsc-csem.org) made it possible to link them to earthquakes that occurred in Kyrgyzstan and Japan (direction "east" relative to Tashkent). Recordings of directional detectors, corresponding to these earthquakes, are shown in Fig. 5.

As can be seen from these figures, the maximum signals are obtained from directional detectors, oriented to east (curve 1), while signals from neighboring detectors (curve 2-NE, and curve 3-SE) are much weaker.

The above presented results indicate the possibility of forecasting the earthquake and defining the direction of its epicenter by measuring the intensity of fluxes of low-energy neutrons and charged particles, exhaled from Earth's crust. However, for a more convincing interpretation of the results and proper identification of the location of the epicenter, more data from similar stations are needed. We believe that creation of a network of such stations is of great importance for improving the reliability of earthquake prediction by the proposed method.

\section{Conclusions}

A new method for earthquake forecasting is proposed, which is based on simultaneous recording of the intensity of fluxes of low-energy neutrons and charged particles by detectors used in nuclear physics. These particles mainly emerge from $\alpha$-radioactive phenomena and partially associated with the radon gas, flowing to the surface during tectonic processes in the Earth's crust. An installation has been created which allows to measure the variations in fluxes of neutrons and charged particles, emanating from the Earth's crust. The upgraded version of the installation with directional detectors can provide the possibility to define the direction of the epicenter. The upgraded 

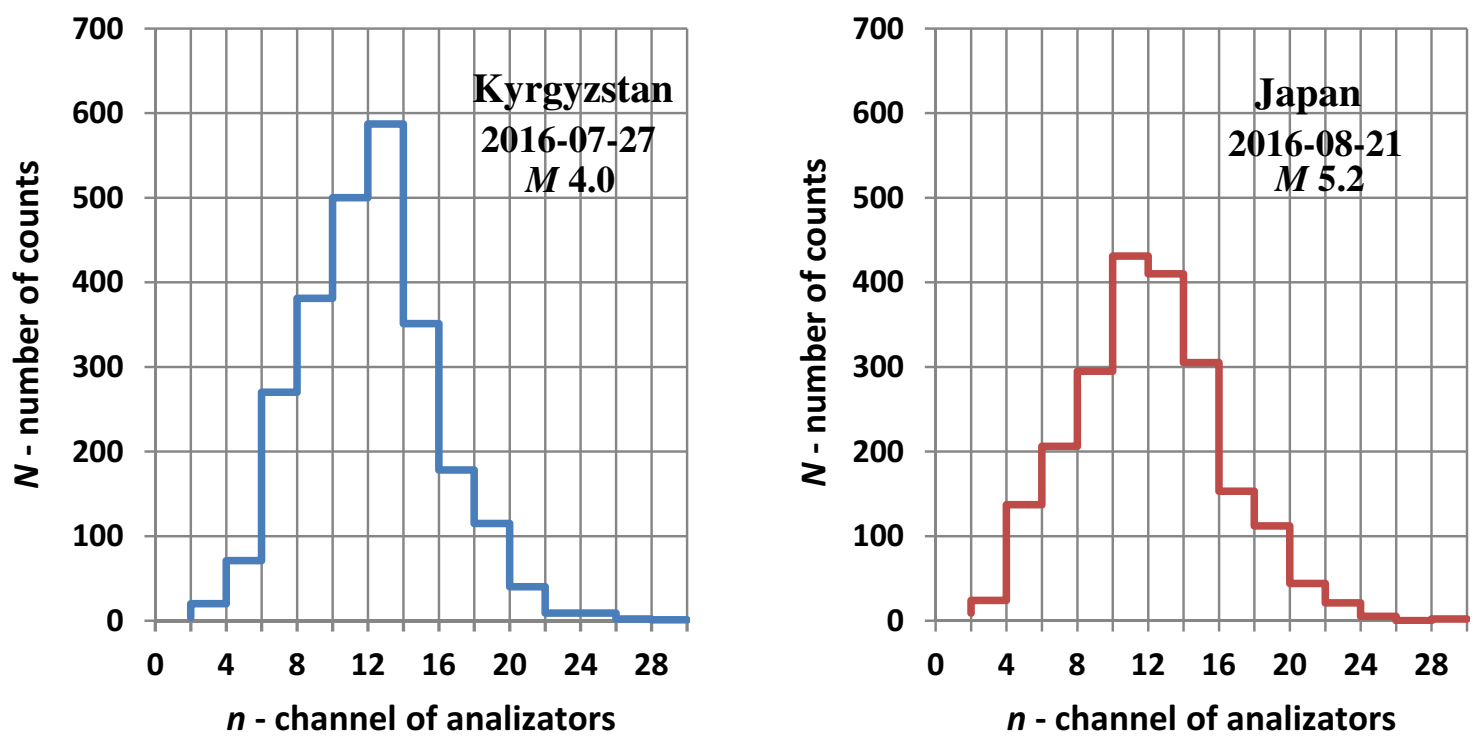

Fig. 4 Distribution of registered signals for 8-10 h before the earthquake in Kyrgyzstan (July 27, 2016, M 4.0) and in Japan (August 21, 2016, $M$ 5.2). The background intensity is taken as zero
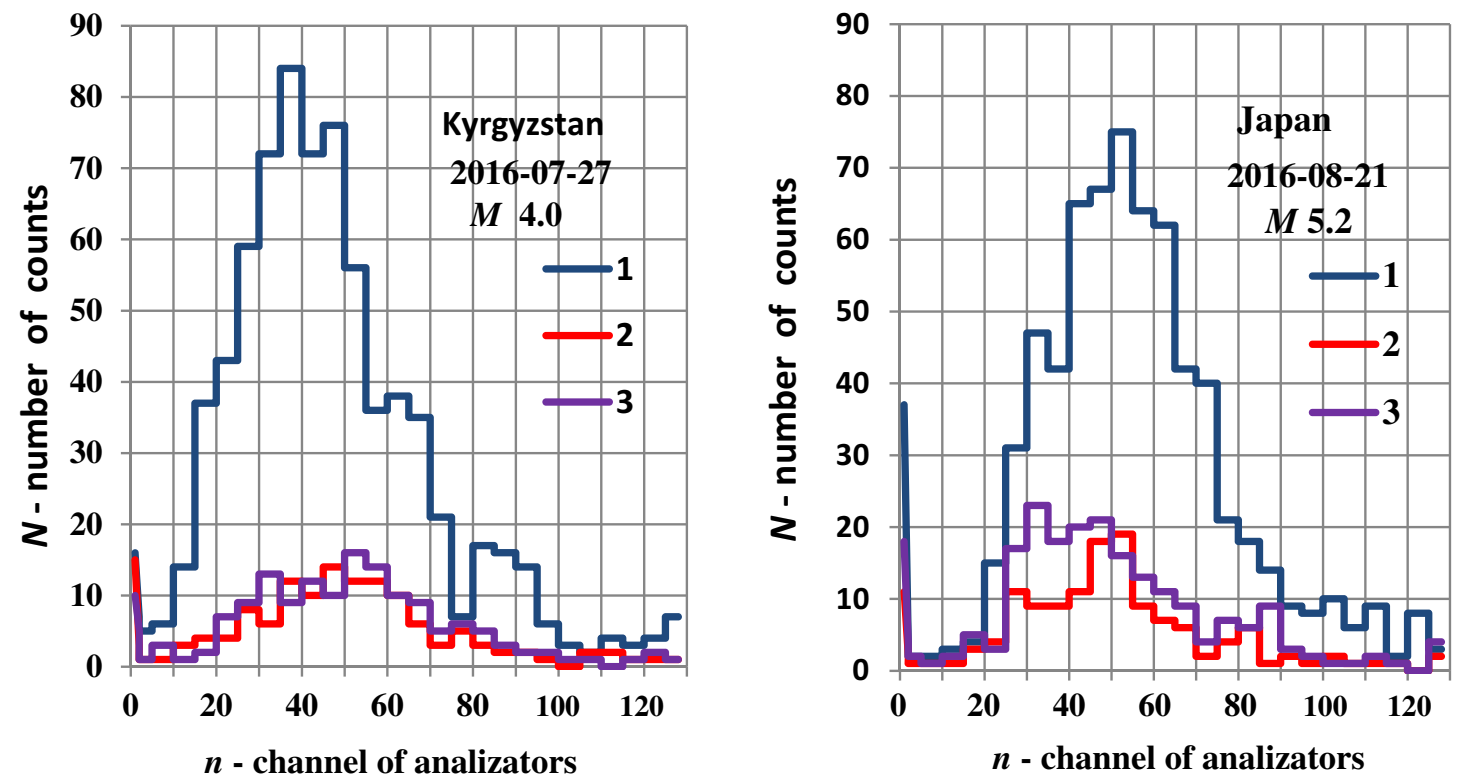

Fig. 5 Distribution of signals from directional detectors, oriented to 1 - east, 2-NE (northeast), 3-SE (southeast), for Kyrgyzstan (July 27, 2016, $M$ 4.0) and Japan (August 21, 2016, $M$ 5.2) earthquakes

installation has been tested with reference to several earthquakes, occurred in 2016. From the obtained results, we conclude that the proposed method is capable of forecasting impending earthquakes by 10 and more hours before the event.

Open Access This article is distributed under the terms of the Creative Commons Attribution 4.0 International License (http://crea tivecommons.org/licenses/by/4.0/), which permits unrestricted use, distribution, and reproduction in any medium, provided you give appropriate credit to the original author(s) and the source, provide a link to the Creative Commons license, and indicate if changes were made.

\section{References}

Antonova VP, Volodichev NN, Kryukov SV, Chubenko AP, Shchepetov AL (2007) Effect of solar eclipses on neutron flux variations at the Earth's surface. Bull Russ Acad Sci Phys 71(7): 1054-1057

De SS, De BK, Bandyopadhyay B, Paul S, Haldar DK, Bhowmick A, Ali R (2010) Effects on atmospherics at 6 and $9 \mathrm{kHz}$ recorded at 
Tripura during the India-Pakistan Border earthquake. Nat Hazards Earth Syst Sci 10(4):843-855

De SS, Bandyopadhyay B, Das TK, Paul S, Haldar DK, De BK, Chattopadhyay G (2011a) Studies on the anomalies in the behavior of transmitted subionospheric VLF electromagnetic signals and the changes in the fourth Schumann resonance mode as signatures of two pending earthquakes. Indian $\mathrm{J}$ Phys 85(3):447-470

De SS, De BK, Bandyopadhyay B, Paul S, De D, Barui S, Das TK (2011b) Studies on the precursors of an earthquake as the VLF electromagnetic sferics. Rome J Phys. 56(9-10):1208-1227

De SS, Paul S, Haldar DK, De D, Kundu AK, Chattopadhyay S, Barui S (2012) Analyses of the effects of several earthquakes on the sub-ionospheric VLFLF signal propagation. J Atmos Sol Terr Phys 81:20-26

European-Mediterranean Seismological Center. http://www.emsccsem.org. Accessed 10 Nov 2016

Hazra P, Barui S, De SS, Paul S (2015) Studies on the influence of two large earthquakes $(M>6)$ upon $9 \mathrm{kHz}$ sferics recorded from Kolkata. Rom J Phys 60(7-8):1218-1224

Hazra P, De SS, Paul S, Guha G, Ghosh A (2017) Thermal anomalies around the time of Nepal Earthquakes $M 7.8$ April 25, 2015 And M 7.3 May 12, 2015. Int J Geotechn Earthq Eng 8(1):58-73

Karia S, Sarkar S, Pathak K, Sharma AK, Ranganath H, Gwal AK (2013) Analysis of space- and ground-based parameters prior to an earthquake on 12 December 2009. Int J Remote Sens 34(21):7779-7795

Kuzhevskij BM, Nechaev OY, Sigaeva EA, Zakharov VA (2003) Neutron flux variations near the Earth's crust. A possible tectonic activity detection. Nat Hazards Earth Syst Sci 3:637-645

Li M, Parrot M (2013) Statistical analysis of an ionospheric parameter as a base for earth-quake prediction. J Geophys Res Space Phys 118(6):3731-3739

Maksudov AU (2015) Patent for useful model. Republic of Uzbekistan, No. FAP 01088 from 04.02.2015

Maksudov U (2016) Monitoring of seismic harbingers for the forecast of earthquakes. Comput Nanotechnol 1:52-61

Maksudov AU, Muminov RA, Shayakubov DB (2015) A setup for detecting earthquake precursors. Instrum Exp Tech 58:130-131
Sarkar S, Tiwari S, Gwal AK (2011) Electron density anomalies associated with $M \geq 5.9$ earthquakes in Indonesia during 2005 observed by DEMETER. J Atmos Sol Terr Phys 73(16):2289-2299

Sarkar S, Choudhary S, Sonakia A, Vishwakarma A, Gwal AK (2012) Ionospheric anomalies associated with the Haiti earthquake of 12 January 2010 observed by DEMETER satellite. Nat Hazards Earth Syst Sci 12(3):671-678

Setyaningsih E, Achmad MS, Novianta MA (2014) Wireless earthquakes feature monitoring based on acceleration and magnetic measurements using MEMS sensor. In: Proceedings of the 1st International Conference on Engineering Technology and Industrial Application 2014, pp 189-192

Silva HG, Bezzeghoud M, Oliveira MM, Reis AH, Rosa RN (2013) A simple statistical procedure for the analysis of radon anomalies associated with seismic activity. Ann Geophys 56(1):R0106

Ulomov VI, Mavashev BZ (1967) A precursor of a strong tectonic earthquake. Dokl Acad Sci USSR Earth Sci Sect 176:9-11

Volodichev NN, Kuzhevskij BM, Nechaev OY, Panasyuk MI, Podorolsky AN, Shavrin PI (2003) Solar-lunar-terrestrial interactions: bursts of neutron emission and seismic activity. Int $\mathbf{J}$ Geomagn Aeron 4(3):251-254

Woith H (2015) Radon earthquake precursor: a short review. Eur Phys J Spec Top 224:611-627

Yuldashbaev TS, Maksudov AU (2010) Development of the method for detecting the earthquake precursors by recording time variations of fluxes of cosmic rays and neutrons. Dokl Acad Sci Rep Uzb (3):37-41 (Reports of Uzbekistan Academy of Sciences. In Russian)

Yuldashbaev TS, Maksudov AU (2012) Installation for measuring the intensity of low energy neutrons and charged particles. Dokl Acad Sci Rep Uzb (2):20-22 (Reports of Uzbekistan Academy of Sciences. In Russian)

Yuldashbaev TS, Muminov RA, Maksudov AU, Shayakubov DB (2012) Studying the stream variations of the charged particles and low energy neutrons. Uzb J Phys 14:144-148 (in Russian)

Zeren ZM, Zhang XM, Shen XH, Sun WH, Ning DM, Ruzhin Y (2014) VLF radio signal anomalies associated with strong earthquakes. In: General Assembly and Scientific Symposium, (URSI GASS), 2014 XXXIth URSI. IEEE 